\title{
Calibration and Evaluation of Fixed and Mobile Relay-Based System Level Simulator
}

\author{
Shahid Mumtaz, Le Thanh Tu, Kazi Saidul, and Atílio Gameiro \\ Institute of Telecommunications, Department of Electronic, Telecommunication and Computer Science, \\ University of Aveiro, Aveiro 3810078, Portugal \\ Correspondence should be addressed to Shahid Mumtaz, smumtaz@av.it.pt
}

Received 7 March 2010; Accepted 30 June 2010

Academic Editor: Sghaier Guizani

Copyright ( $\odot 2010$ Shahid Mumtaz et al. This is an open access article distributed under the Creative Commons Attribution License, which permits unrestricted use, distribution, and reproduction in any medium, provided the original work is properly cited.

\begin{abstract}
Future wireless communication systems are expected to provide more stable and higher data rate transmissions in the whole OFDMA networks, but the mobile stations (MSs) in the cell boundary experience poor spectral efficiency due to the path loss from the transmitting antenna and interference from adjacent cells. Therefore, satisfying QoS (Quality of Service) requirements of each MS at the cell boundary has been an important issue. To resolve this spectral efficiency problem at the cell boundary, deploying relay stations has been actively considered. As multihop/relay has complex interactions between the routing and medium access control decisions, the extent to which analytical expressions can be used to explore its benefits is limited. Consequently, simulations tend to be the preferred way of assessing the performance of relays. In this paper, we evaluate the performance of relay-assisted OFDMA networks by means of system level simulator (SLS). We consistently observed that the throughput is increased and the outage is decreased in the relay-assisted OFDMA network, which is converted to range extension without any capacity penalty, for the realistic range of values of the propagation and other system parameters investigated.
\end{abstract}

\section{Introduction}

Computer simulations are often used to predict the performance of cellular networks. Typically, link level simulators are used to model the link between the base and mobile station, while system level simulators model the entire network. To most accurately predict the performance of cellular networks, a system level simulator, which includes the performance of link between each base and mobile station, should be used. Unfortunately, the computational complexity of such simulators makes this impractical. An alternative technique consists of separately simulating the link and system components with an interface or mapping to combine the results.

The concept of relaying in system level simulator has been a well-known technique for transmission of signals over very long distance. Research results on relay channels can be found in the 1970s [1, 2]. Recently, due to the explosion of interests in ad hoc networks and the research activity on the fourth generation $(4 \mathrm{G})$ wireless communication systems, the concept of relay networks was again brought to the mainstream of research in mobile communication academy and industry $[3,4]$. It is unlikely that $4 \mathrm{G}$ mobile systems can cover the same service area without additional infrastructure. The problem of path loss will prohibit the base station from communicating with users far away [5].

One way to overcome those problems is to use relays to extend the coverage area in cellular networks. Relay is introduced as a method to solve the problems. The norm Relay traditionally appears in ad hoc networks, where two nodes communicate indirectly via other nodes, which are called relay nodes. According to [6], three types of Relay Stations (RSs) are defined: fixed, nomadic, and mobile. The relays are in most cases built in, owned, and controlled by service provider. An RS is not directly connected to wire infrastructure and has the minimum functionality to support multihop communication. Multihop-based network may also improve system performance when cooperation relay technique is put to use. This is accomplished by sending information simultaneously via multiple different 
paths and combining the received information at the side of the receiver [7]. Two concepts of how to integrate multihop communication into IEEE 802.16 standard are presented in [8]. The first concept follows a centralized approach, where the BS (base station) has full control over the relay-enhanced cell and RS may be very simple. The second concept follows a semidistributed approach, where RS coordinates the associated Subscriber Stations (SSs) itself. In the second case, the MAC protocol complexity of RS is comparable to BS. In this paper we evaluate the performance of relay-assisted OFDMA network by mean of system level simulator which include both fixed and mobile relays by using centralized approach. Different type of mobile relays are studied in the literature. Typically mobile relays are classified into two categories. (1) Dedicated Mobile (a mobile relay that is fitted on a moving vehicles, which provides coverage in a designated area. (2) User terminal is elected to provide relaying functionalities to a number of other user terminals. In this paper, we only consider type 2, where mobile user also configure as a mobile relay terminal.

The rest of the paper is organized as follows. Section 2 explains the system level simulator, its architecture, traffic models, scheduling, and link adaptation and SINR modelling. Section 3 explains the enhanced system level simulator with relay capabilities, deployment of mobile and fixed relays, it advantage and disadvantages, and channel model. Section 4 presents the performance metrics and simulation results followed by the conclusion in Section 5 .

\section{System Level Simulator}

Typically, network simulations are divided into two parts: link and system level simulations (SLS) as shown in Figure 1. Although a single simulator approach would be preferred, the complexity of such simulator (including everything from transmitted waveforms to multicell network) is far too high with the required simulation resolutions and simulation times. Also, the time granularity of both domains of simulation are symmetrically different: at link level, bit transmissions are at the order of milliseconds, while at the system level traffic and mobility models require time intervals of some tens of seconds to minutes. Therefore, separate link and system level simulations are needed.

Typically, the link level simulator is able to predict the receiver Frame Erasure Rate/Bit Error Rate (FER/BER) performance, taking into account channel estimation, interleaving, and decoding and is needed to build a model for the system level simulator, which is needed to model a system with a large number of mobile and base stations and the algorithms operating in such a system. Because the simulation is divided into two parts, a method to interconnect them and a proper interface has to be defined. System performance evaluation of a given mobile wireless access technology requires simulations that carefully captures the dynamics of a multipath fading environment, the architecture of the air interface, mobile stations behaviour (in terms of mobility) and the types of applications used (properly defined traffic models). Also, since system level results depend intrinsically on the simulation scenario (propagation and interference environments, number and distribution of users within the cells) it is important that the assumptions and parameters used in the analysis be reported jointly with performance results. This procedure is fundamental for the correct validation of the results obtained and for benchmarking against other proposed scenarios for different wireless systems.

In SLS, two different types of simulations can be performed: combined snapshot-dynamic mode or a fully dynamic mode. In fully dynamic mode, mobility and handover are enabled and path loss, fast and slow fading recomputed every transmission time interval (TTI). In combined Snapshot dynamic mode, mobility and handover are not enabled. Mobiles are randomly deployed in every TTI, path loss and slow fading are computed once at the beginning of each TTI. We consider an IEEE 802.16 cellular system consisting of 19 cells, with six cells in the first tier and twelve cells in the second tier, surrounding the central cell. Each cell includes three 120-degree sectors, that is, 57 sectors in total are simulated. All the simulation results are collected from the three central sectors in the central cell, with the other 54 sectors serving as interferers.

Logical diagram of SLS is shown in Figure 2. The SLS interfaces with the link level simulator through look up tables (LUTs) as input to the simulator. Several propagation and traffic models are available, and the simulator computes the entire channel losses (slow and fast fading), thereby ensuring accuracy in the system level parameters computed. The outputs are the parameters that usually characterize packet transmissions: Throughput, BLER, Packet Delay, and so forth.

The traffic generation block contains real (i.e., VoIP, WWW) and nonreal (i.e., NRTV, FTP) time service traffic models with full queue as shown in Figure 3.

Handover block, as the name suggests, includes the handover algorithm.

The radio resource management block comprises a call admission control algorithm to regulate the operation of the network, a link adaptation algorithm to select the appropriate parameters in function of the current radio conditions, and a scheduler that decides how to allocate the appropriate resources, based on the service type, the amount of data, the current load in the cell, and so forth. The power control block contains mechanisms to provide similar service quality to all communication links despite the variations in the channel conditions. The interference block determines the average interference power received by central base station, that is, intercell interference.

Finally, the computations of system level metrics block returns the network results such as Service Throughput (average spectral efficiency), Block Error Rate, and Packet Delay. The mobility block models mobile movement in indoor, urban, and rural environments. Parameters associated with mobility include speed, probability to change speed at position update, probability to change direction, and the decorrelation length. The propagation block models path loss, slow fading and fast fading. Channel models for indoor environments, outdoor urban, and rural environments are 


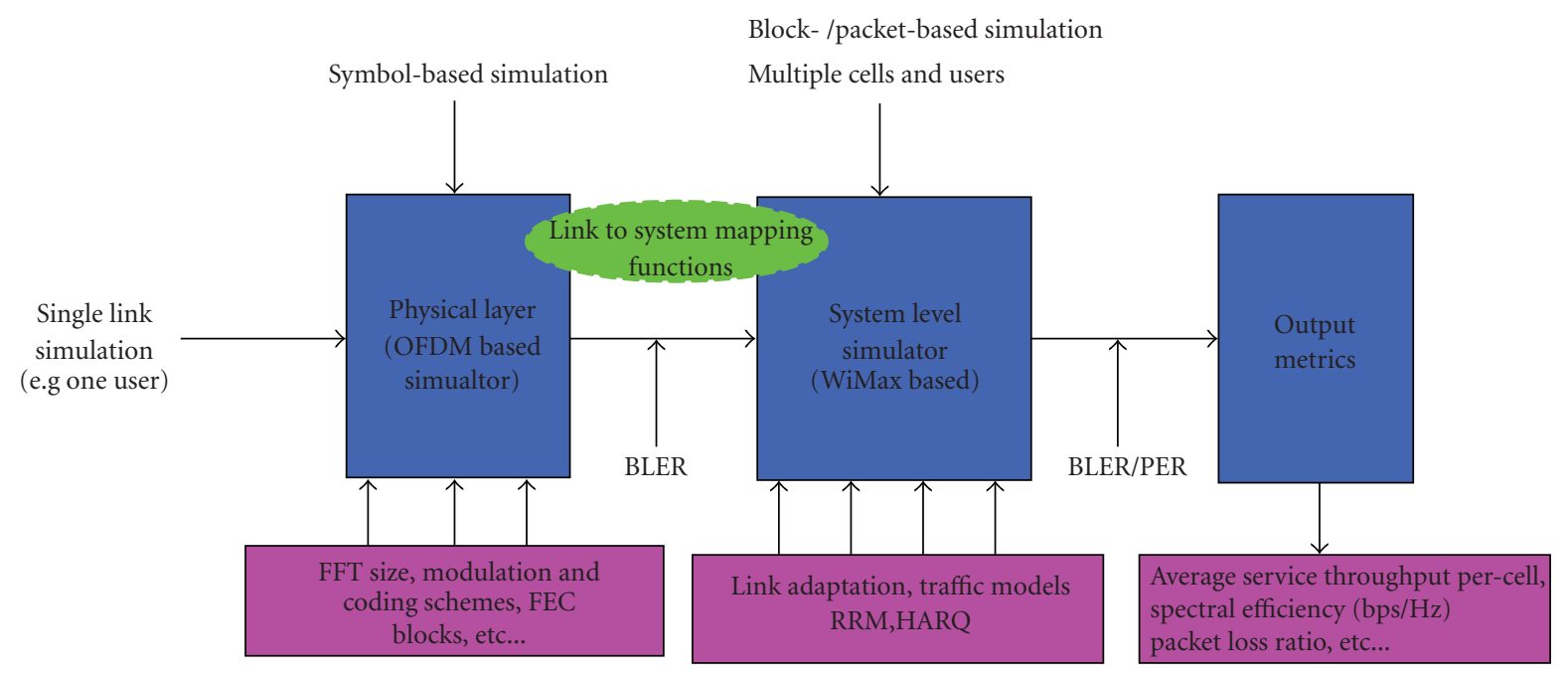

Figure 1: Network simulator.



FIgURE 2: Logical SLS.

available. The scheduler mechanism will generate the arrival process of the users, according to a Poisson arrival process.

The objective of Call Admission Control is to regulate the operation of a network in such a way that ensures the uninterrupted service provision to the already existing connections and at the same time accommodates in an optimum way the new connection request. The scheduler decides how to allocate the appropriate resources, based on the service type, the amount of data, the load on the common, and shared channels, the current loading in the cell and the radio performance of each type of transport channel. Link adaptation can be considered as a component of Dynamic Channel Assignment. With the power control mechanism, similar service quality is provided to all communication links despite the variations in the channel conditions, which means larger proportion of the total available power is consumed for the bad channel conditions. Handover is common to all dynamic system level simulators and required to maintain link quality at the cell boundaries. Simulation map description of the cellular map, which includes the cell descriptions, base station locations, and the manner in which it will model mobile movement at the system boundaries. Simple ARQ is employed for nonreal time services. Dynamic Channel Allocation algorithm provides extra performance, but is not a pivotal element in the simulator.

2.1. Frame Structure. Figure 5 illustrates the IEEE802.16e TDD frame structure. Although mobile WiMAX standard 


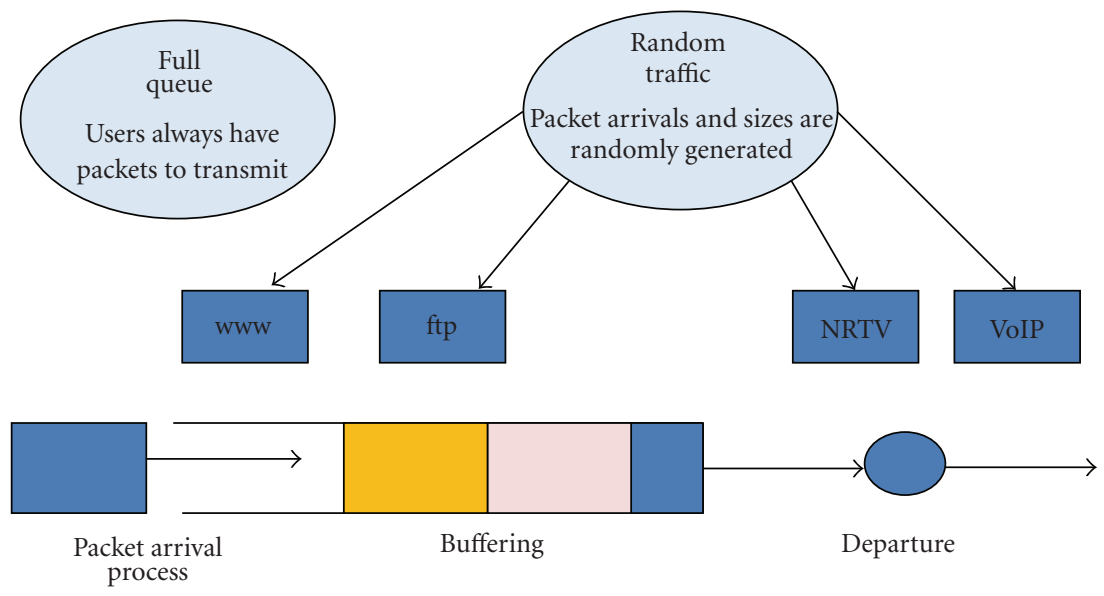

Figure 3: Traffic models.

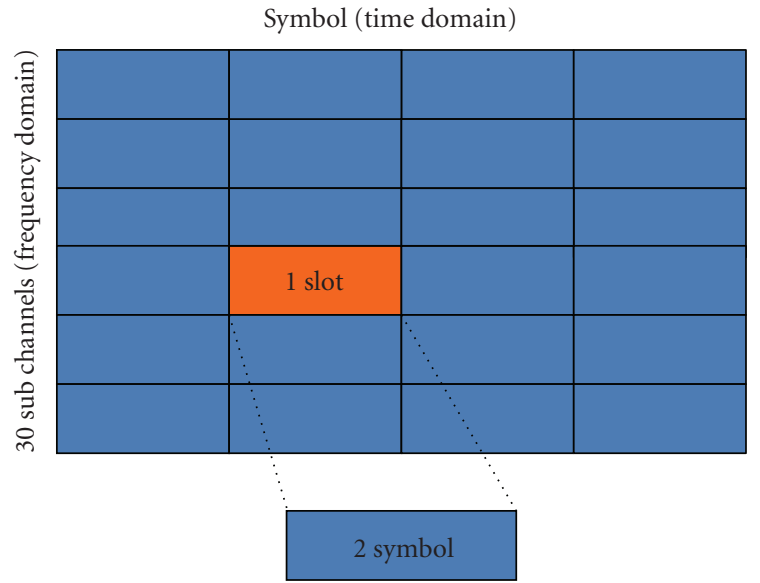

FIgURE 4: Resource allocation map (RAM).

support both frequency division duplexing (FDD) and time division duplexing (TDD), only the TDD mode is being supported by the system profiles designed by the WiMAX forum for equipment compliance and interoperability. Also, TDD offers some advantages over the FDD mode such as the support of asymmetrical data rates in UL and DL and also the fast estimation of the downlink channel in the UL transmission. In the frame, resources are available in two domains: frequency (subcarriers) and time (OFDM symbols). The OFDM symbols available for data transmission and the subcarriers constitute distinct logical slots. Different types of logical channels are available, depending of the type of channel model used.

The standard defines two basic types of generic channel modes: channel modes that are intrinsically diverse in frequency called diversity subcarrier permutation modes and the channel modes that make use of the frequency selective nature of the radio channel. In this paper, we focus primarily on the so-called Partially Usage Subcarrier Channelization (PUSC) channel mode. In PUSC mode, every subchannel
TABle 1: (a) Theoretical throughputs. (b) MCS levels.

(a)

\begin{tabular}{lc}
\hline MCS & Theoretical throughput (Mbps) \\
\hline QPSK 1/2 & 4.32 \\
QPSK 3/4 & 6.48 \\
16 QAM 1/2 & 8.64 \\
16 QAM 3/4 & 12.96 \\
64 QAM 1/2 & 12.96 \\
64 QAM 3/4 & 19.44 \\
\hline
\end{tabular}

(b)

\begin{tabular}{lcc}
\hline MCS level & MCS & SINR threshold $(\mathrm{dB})$ \\
\hline 1 & QPSK 1/2 & 9.42 \\
2 & QPSK 3/4 & 11.8 \\
3 & 16 QAM 1/2 & 16.6 \\
4 & 16 QAM 3/4 & 18.9 \\
5 & 64 QAM 1/2 & 22.6 \\
6 & 64 QAM 3/4 & 24.4 \\
\hline
\end{tabular}

is available for allocation in the cell. The data subcarriers are randomly allocated along the whole spectrum of the FFT. In the PUSC mode, the small unit of allocation is the slot. A group of slots defines a burst in the radio frame. A burst can be assigned to more than one user provided the same modulation and coding scheme is followed in the transmission of all packets allocated to it. PUSC schematic description of the Resource Allocation Map (RA) in the WiMAX System Level Simulator is given by Figure 4. Total number of symbol available in one OFDM frame are 48, as we consider only downlink transmission so our frame contain 30 OFDM symbol $(35-5=30,5$ symbols for control information, e.g., Preamble, FHC and remaining symbol are used for uplink transmission).

According to PUSC configuration, 1 slot contain 2 OFDMA symbols in time domain and 720 data subcarriers in frequency domain. So, we have total 30 logical subchannels 


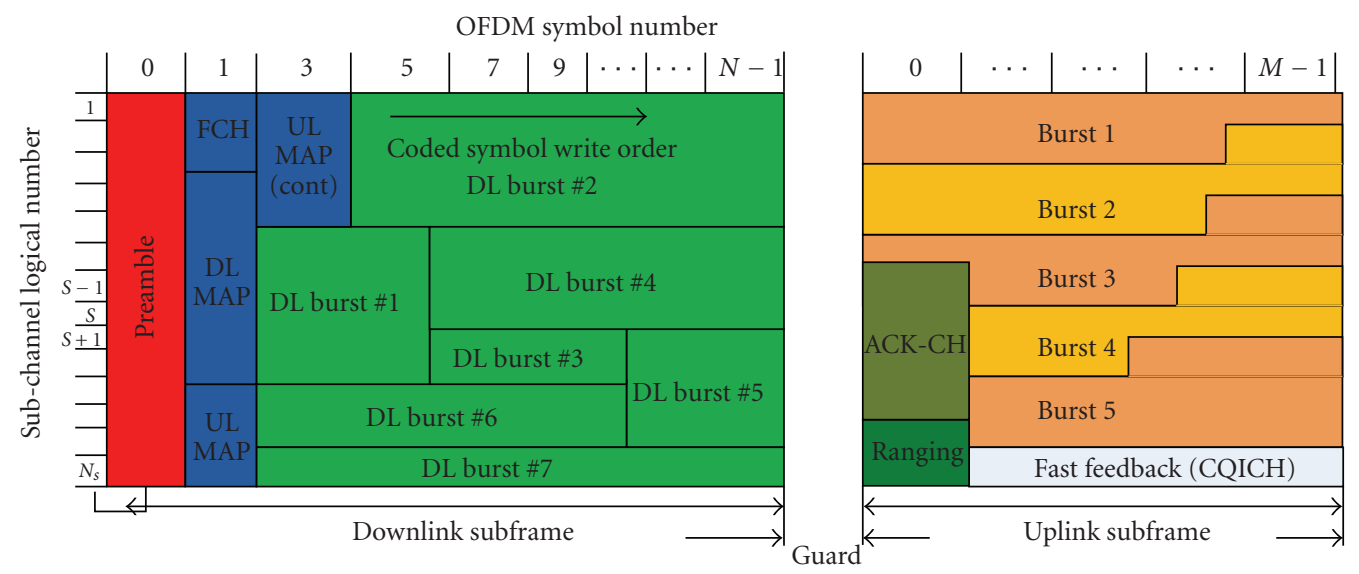

Figure 5: WIMAX frame structure.

that contain 24 subcarriers each $(30 * 24=720)$ in frequency domain.

To calculate the theoretical throughput of each coding and modulation schemes using PUSC configure, we illustrate following numerical example.

Consider that we have,

$$
\begin{aligned}
& 1 \text { slot }=2 \text { OFDM symbols, } \\
& 1 \text { subchannel }=24 \text { data subcarriers, } \\
& \text { downlink symbol }=30 \text { symbol, } \\
& \text { frame length }=5 \mathrm{~ms},
\end{aligned}
$$

then total number of slot in one OFDM frame is $(30 / 2) * 30=$ 450 slots, and $48(2 * 24)$ subcarriers. For example, each slot is modulated with 64 QAM and $3 / 2$ coding rate then, $48 *$ $\log _{2}(64) * 3 / 4=216$ bit.

Peak data rate $=216 * 450$ (slots) $/ 5 \mathrm{~ms}=19440000$ bit per second or 19.44 Mbps as shown in Table 1(a).

2.2. Scheduling. Although the signalling messages and control channels that are required for resource allocation are defined by the standard, the design of the resource allocation policy and scheduling mechanisms are intellectual property, leaving it for manufacturers as a means for equipment differentiation. Nevertheless both scheduling and optimization of radio resource usage are two fundamental aspects that operators need to address so as to maximize network utility in an era where spectral resources are at a premium. In this paper, we have used the Max C/I scheduler [9]. The Max C/I scheduler opportunistically assigns resources to the user with the highest channel gain in the following way. At the beginning of each radio frame $n$, the mobile with the best channel quality indicator (CQI) is scheduled for transmission. The user is chosen according to the following rule

$$
k(n)=\arg \max _{i \in\{1, \ldots, K\}} R_{i}(n), \quad n=0,1,2, \ldots,
$$

" $k$ " is the user, $R_{i}$ is the throughput achieved for the MCS scheme as shown in Table 1 , and " $n$ " is the number of frame transmitted. The max C/I scheduler is very effective in resources allocation in terms of capacity maximization.
2.3. Link Adaptation. The proper transmission mode (modulation and code scheme used-MCS) is defined by the link adaptation method. We have considered $6 \mathrm{MCS}$ schemes encompassing QPSK, 16 QAM, and 64 QAM modulation schemes and the Convolutional encoder, according to the profiles envisioned by the WiMAX forum. For each selected user in each allocated resource, the MCS scheme to be used is chosen according to the following method:

$$
i=\max _{i \in \mathrm{MCS}_{\mathrm{set}}}\left[\left(R_{i}\left(1-\mathrm{BLER}_{i}\right)\right],\right.
$$

where $\mathrm{MCS}_{\text {set }}$ is the set of modulation and coding schemes, $R_{i}$ is the throughput achieved for the MCS scheme as shown in Table 1, and BLER $i$ is the predicted BLER for the MCS scheme. Adaptive modulation and coding (AMC) is utilized, and a possible example of the modulation and coding schemes (MCSs) given in 802.16 is listed in Table 1 (b), as the bit error rate requirement is set to $10^{-5}$.

In the case that the perceived SINR at the receiver cannot satisfy the lowest SINR threshold, that is, $0 \mathrm{~dB}$, no service can be provided, which is denoted as MCS level 0 .

2.4. SINR Modelling. In the simulations, a wideband SISO channel model is implemented by a six tapped delay model shown in Table 2, according to the Ped B $3 \mathrm{Km} / \mathrm{h}$ channel model from [10].

The narrowband fading channel is generated by a Jake's model where the carrier frequency and the speed are used to define the statistics of the fading [11]. Slow fading is modelled according to a log normal distribution. Spatial shadowing correlation between mobiles and base stations is implemented. The shadowing $\mathrm{SH}_{j}(x, y)$ in $\mathrm{dB}$ between one MS at position $(x, y)$ and BS $j$ is given by

$$
\mathrm{SH}_{j}(x, y)=\sqrt{0.5}\left[\left(F_{o}(x, y)+F_{j}(x, y)\right],\right.
$$

where $F_{o}(x, y)$ and $F_{j}(x, y)$ are spatial functions with a Gaussian distribution with zero mean and standard deviation $(\sigma)$ in $\mathrm{dB}$, generated using the method described in [12]. They have a spatial correlation given by

$$
R(d)=\exp ^{[-\ln (2)(d / \text { DecorreLength })]},
$$


TABLE 2: Multipath channel model for ITU Ped B.

\begin{tabular}{lccccccc}
\hline Channel Model & & Path 1 & Path 2 & Path 3 & Path 4 & Path 5 & Path 6 \\
\hline \multirow{2}{*}{ ITU Ped. B } & Path Power (dB) & 3.92 & 4.82 & 8.82 & 11.92 & 11.72 \\
& Delay (ns) & 0 & 200 & 800 & 1200 & 27.82 \\
& & & & & 2300 & 3700 \\
\hline
\end{tabular}

where $d$ is the distance between two points in the network layout and DecorrLength is the shadowing decorrelation length in meters.

Using the aforementioned channel models, the SINR of each OFDM subcarrier is computed according to the following expression $[13,14]$

$$
\gamma_{k}=\frac{I_{\mathrm{or}}}{I_{\mathrm{oc}}+N_{o}} \cdot \frac{N_{\mathrm{used}}}{N_{d}+\mathrm{PDR} \cdot N_{p}} \cdot H_{k}
$$

where $N_{\text {used }}$ is the total number of subcarriers, PDR is the pilot-to-data subcarrier power ration, $N_{d}$ is the number of data subcarriers per OFDM symbol, $N_{p}$ is the number of pilot subcarriers per OFDM symbol, $N_{o}$ is the receiver thermal noise power and $I_{\mathrm{oc}}$ is the other-cell noise power density (assumed spatially and temporally uncorrelated). The gain of the $H_{k}$ th subcarrier is given by

$$
H_{k}=\left|\sum_{p=1}^{N_{\text {paths }}} M_{p} A_{p} e^{j \theta} e^{-2 \pi f_{k} T_{p}}\right|^{2}
$$

where $p$ represents the multipath index for Ped B channel, $A_{k}$ is the amplitude value corresponding to the long-term average power for the $p$ th path, $f_{k}$ is the relative frequency offset of the $k$ th subcarrier of the PUSC specific subchannel, and $T_{p}$ is the relative time delay of the $p$ th path.

2.5. Link Level Interface. In the receiver, postprocessing of the signals received from the serving and interfering Base stations (BSs) is performed. For a Single-Input-SingleOutput (SISO) architecture and a matched filter in the receiver, the received signal at the $k$ th subcarrier for the target user is computed according to

$$
\begin{aligned}
Y^{(0)}[k]= & \sqrt{P_{\text {slot }}^{(0)} P_{\text {loss }}^{(0)}} H^{(0)}[k] X^{(0)}[k] \\
& +\sum_{j=1}^{N} \sqrt{P_{\text {slot }}^{(j)} P_{\text {loss }}^{(j)}} H^{(j)}[k] X^{(j)}[k]+N^{(0)}[k],
\end{aligned}
$$

where $N$ is the number of interferers, $P_{\text {slot }}^{(j)}$ is the transmit power per slot for the $j$ th cell, $P_{\text {loss }}^{(j)}$ is the dependent path loss, including shadowing and antenna gains/losses, $H^{(j)}[k]$ is the channel gain for the desired mobile station (MS) from the $j$ th cell and for the $k$ th subcarrier, $X^{(j)}[k]$ is the transmitted symbol by the $j$ th cell at the $k$ th subcarrier, $N^{(0)}[k]$ is the thermal noise at the received subcarrier, modelled as AWGN with zero mean and variance $\sigma_{n}^{2}$.

The transmission of a coded block over different sets of subcarriers results in a number of SINR measures that equals the number of subcarriers sets, which can be quite high.
Hence, data compression is mandatory. The coded symbol SINR in subcarrier $k$ is given by

$$
\operatorname{SINR}^{(0)}[k]=\frac{P_{\text {slot }}^{(0)} P_{\text {loss }}^{(0)}\left|H^{(0)}[k]\right|^{2}}{\sum_{j=1}^{N} P_{\text {slot }}^{(j)} P_{\text {loss }}^{(j)}\left|H^{(j)}[k]\right|^{2}+\sigma_{n}^{2}} .
$$

The set of coded symbols SINRs are mapped onto a single value named the Effective SINR value.

\section{ARQ Block Decoding}

The SINR of each ARQ block is calculated as the average SINR value of the slots in which the ARQ block is fitted in. EESM [15] is used to compute this average SINR ARQ $_{4}$ value. Then, if the ARQ block is a retransmitted version of a previous ARQ block, the SINR of the ARQ block is recalculated.

This is due to the HARQ mechanism. In this case, a CC (Chase Combining) model is used to perform this task [16]:

$$
\mathrm{SINR}_{\mathrm{eff}}=\sum_{r=0}^{R} \operatorname{SINR}_{\mathrm{ARQ}}(t-r),
$$

where $R$ denotes the number of retransmissions. Once the SINR $_{\text {eff }}$ of each ARQ block is calculated, the BS decides if this block was transmitted with an error or not. To take this

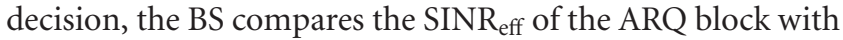

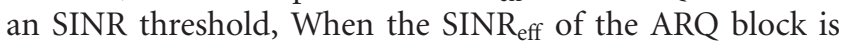
larger than the threshold, the BS assumes that the packet is transmitted successfully; otherwise, the BS assumes that the packet is erroneous, and it asks for a retransmission.

Note that link-level simulations and a random variable uniformly distributed between 0 and 1 is used to select the SINR threshold. In this way, the randomness of the decoding process is captured. Figure 6(a) illustrates this decision process. If the packet is not successfully transmitted after a maximum number of retransmissions $(\max =3)$, the ARQ block is discarded.

The mechanism of Effective SINR is shown in Figure 6(b). This value can be used to match AWGN Look-Up Tables (LUTs). The EESM [15] expression determines how the effective SINR is obtained from the multiple SINR's on the different subcarriers

$$
\mathrm{SINR}_{\mathrm{eff}}=-\beta \ln \left(\frac{1}{P} \sum_{p=1}^{P} e^{-\operatorname{SINR}_{p} / \beta}\right),
$$

where $P$ is the total number of subcarriers, $\operatorname{SINR}_{p}$ is the SINR per subcarriers $P, \beta$ is depending upon each MCS and is to be optimized for every link mode (MCS) based on link level simulation results. 


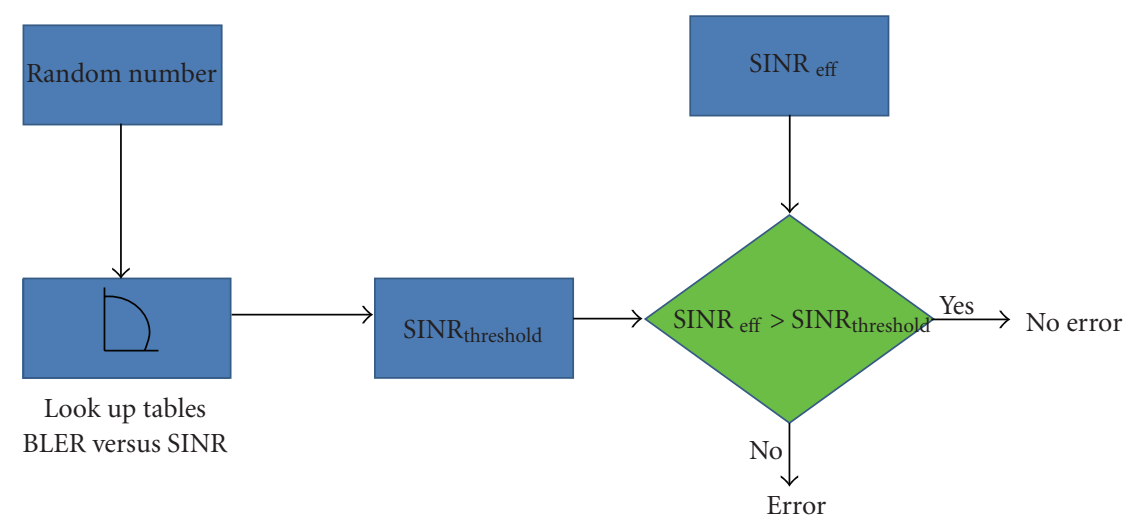

(a)

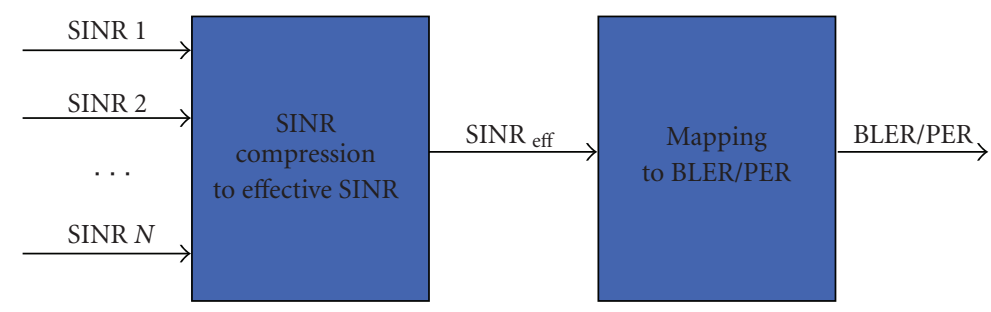

(b)

FIGURE 6: (a) ARQ acceptance process. (b) Effective SINR mapping.

The following steps are performed to obtain beta $(\beta)$ value from link level simulation.

(1) Generate an AWGN curve for a specific MCS level.

(2) Measure the SINR per-tone (subcarrier) values for the same MCS level using the desired channel model (for instance Ped B). Many channel realizations are required and SINR per-tone values should be converted to one scalar value to represent the channel SINR using EESM formula in (10).

(3) Compare the two values gained from the previous steps $\left(\right.$ SINR $_{\text {EESM }}$ and SNR $\left._{\text {AWGN }}\right)$. The comparisons for many SNR-pair values will yield a mean squared difference for a given beta value. Figure 7 shows the abstraction of beta $(\beta)$. The beta value that gives the minimum difference is selected as the optimal value

$$
\beta=\arg \min _{\beta}\left\|\operatorname{SNR}_{\mathrm{AWGN}}-\operatorname{SINR}_{\mathrm{eesm}}(\beta)\right\| .
$$

\section{Enhanced Relay-Based System Level Simulator}

In this section, we enhanced our system level simulator (SLS) with relay capabilities.

4.1. System Overview. A network of seven clusters with four hexagonal cells in each cluster is considered in this paper. Similar to current cellular networks, the BS is located in the centre of the hexagonal cell. Six fixed relays are placed in each cell as new network elements enhancing the cellular infrastructure. Each fixed relay is located on the line that connects the centre of the cell to one of the six cell vertices, and it is $2 / 3$ away from the centre (BS) as shown in Figure 8.

Relay placement algorithm is as follows.

(i) First relay has been placed on the open area with coverage radius of " $x$ " and its axis on the space is $(x+y, x+y)$, where " $y$ " $(y \geq x)$ is constant.

(ii) 2 nd relay has been placed at distance of " $z$ " from the centre point of 1 st relay node at $(x+y, z)$.

(iii) 3rd relay placed at $(z, x+y)$, 4 th at $(z, z)$, and so on

$$
\begin{gathered}
{\left[\begin{array}{cccc}
x+y & x+y & z & z \\
x+y & z & x+y & z
\end{array}\right] \quad \text { if } x+y=a,} \\
\left(S_{1 j}, S_{2 j}\right)=\left[\begin{array}{cccc}
a & a & z & z \\
a & z & a & z
\end{array}\right] .
\end{gathered}
$$

4.2. Mobile Relay. Mobiles are randomly deployed in hexagonal cells around the BS and fixed relay as shown in Figure 8. To select which mobile becomes mobile relay station (MRS), we follow the following approach. $\mathrm{Nr}$ is the total number of mobile deployed in the cell and then we generate a random variable that takes the value 0 or 1 . If mobile gets 1 , then it acts as mobile relay otherwise as user terminal

$$
N_{\mathrm{MR}}=\sum_{i=1}^{N_{T}} I_{i},
$$




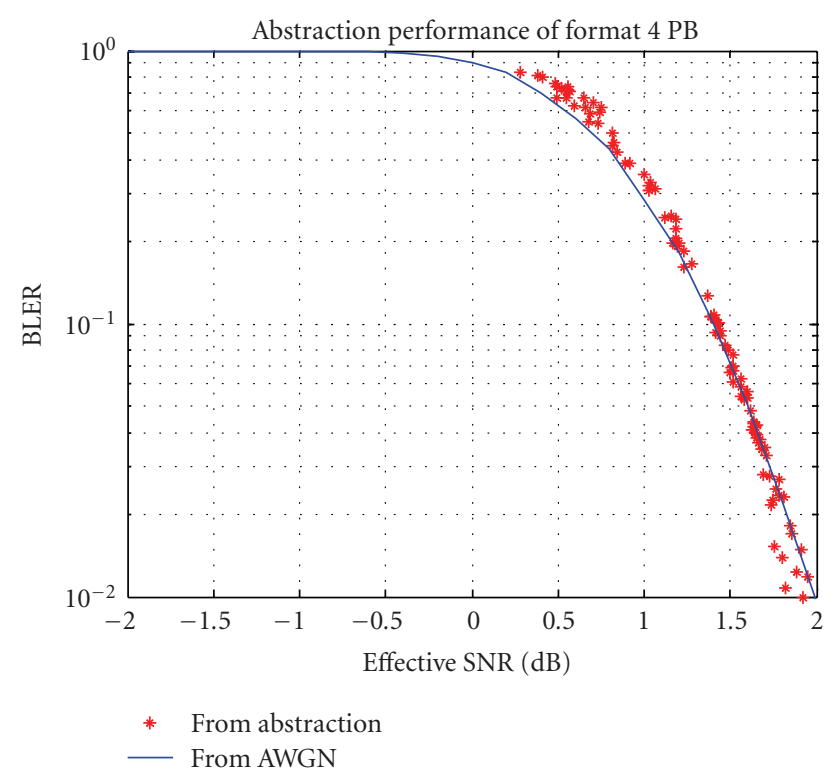

Figure 7: Abstraction of beta $(\beta)$ in Ped B channel.
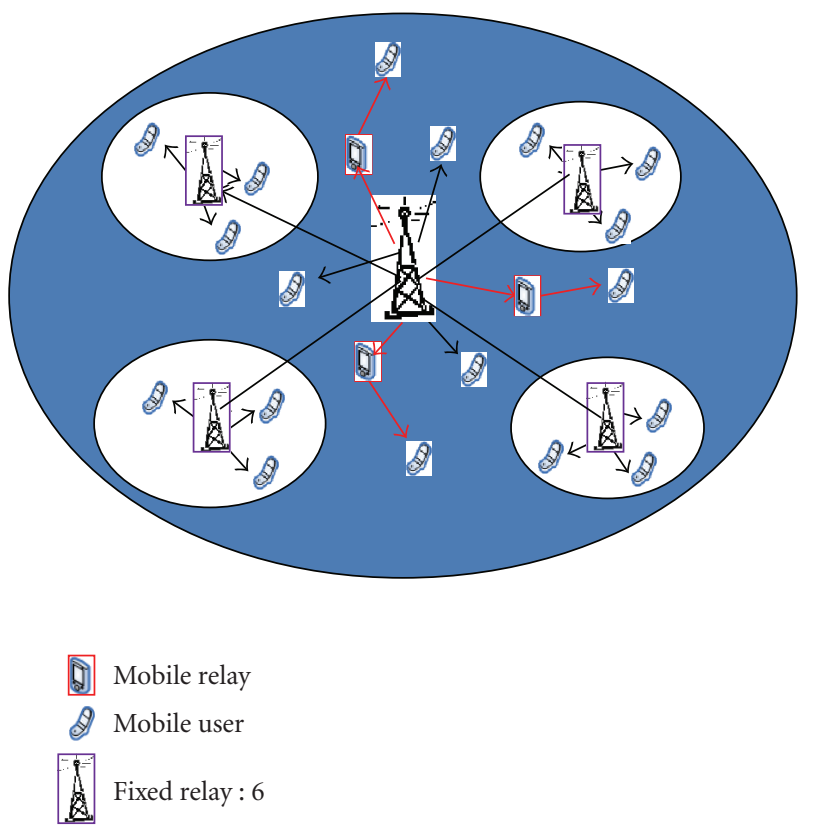

FIgURE 8: Cellular layouts for fixed and mobile relay nodes.

where $N_{T}$ is the total number of mobiles and $I_{i}$ is the random binary variable that takes value 1 if mobile $i$ is used as relay and value 0 if used as user terminal

$$
I_{i}= \begin{cases}1, & \text { with prob } p, \\ 0, & \text { with prob } 1-p,\end{cases}
$$

where $p$ is the parametrizable.

4.3. Fixed Relay. The FRSs (Fixed Relay Stations) are less constrained by energy consumption. The use of FRS eases
TABLE 3: Values of constants.

\begin{tabular}{lccc}
\hline Model parameter & Terrain A & Terrain B & Terrain C \\
\hline $\mathrm{a}$ & 4.6 & 4.0 & 3.6 \\
$\mathrm{~b}$ & 0.0075 & 0.0065 & 0.005 \\
$\mathrm{c}$ & 12.6 & 17.1 & 20 \\
\hline
\end{tabular}

the problem of RS selection since fewer numbers of FRS are deployed compared to MRS. The main disadvantage of using FRS is the infrastructure's cost as compared to MRS.

4.4. Mobile Relay. The main advantage of a relaying system using MRS would be the low deployment/maintenance cost, since other user's terminals can potentially act as relays. If there are a large number of idles MS, there are more choice to select a MRS that can optimize the system performance. For example, a relayed user can choose a MRS with whom it experiences a LOS (Line of Sight) link. If the source terminal and the destination terminal are close to each other, they can communicate directly without sending their traffic through the BS as it is in conventional cellular networks. It is obvious that the relaying opportunity depends strongly on the user's density. Relaying through other users terminals can considerably decrease the battery life of the mobile relay station.

\subsection{Radio Propagation Model}

4.5.1. Path Loss Model. Path loss estimation channel model used in this simulation was developed by Stanford University and was picked up its suitability for fixed broadband wireless access [17]. The basic path loss equation with correction factor is calculated as

$$
\mathrm{PL}=A+10 \gamma \log _{10}\left(\frac{d}{d_{o}}\right)+X_{f}+X_{h}
$$

where $d$ is the distance between the transmitter and the receiver antennas in meter, $d_{o}=100 \mathrm{~m}$. The other parameters are defined as

$$
\begin{gathered}
A=20 \log _{10}\left(\frac{4 \pi d_{o}}{\lambda}\right), \\
\gamma=a-b h_{b}+\frac{c}{h_{b}},
\end{gathered}
$$

where $h_{b}$ is the base station height above the ground in meters and constant $a, b$, and $c$ are the given in Table 3 . The correction factor for the operating frequency and for SS height are given as

$$
\begin{gathered}
X_{f}=6.0 \log _{10}\left(\frac{f}{2000}\right), \\
X_{h}=-20 \log _{10}\left(\frac{h_{r}}{2}\right),
\end{gathered}
$$

where $f$ is the frequency in $\mathrm{MHz}$ and $h r$ is the SS antenna height above the ground in meter. 
TABLE 4: Channel between BS and FRS/MRS.

\begin{tabular}{lcccc}
\hline & BS & FRS & MRS & UT \\
\hline BS & NA & ITU Pedestrian B & ITU Pedestrian B & ITU Pedestrian B \\
FRS & Perfect & NA & ITU Pedestrian B & ITU Pedestrian B \\
MRS & ITU Pedestrian B & ITU Pedestrian B & NA & ITU Pedestrian B \\
UT & ITU Pedestrian B & ITU Pedestrian B & ITU Pedestrian B & NA \\
\hline
\end{tabular}

4.5.2. Shadowing Model. We are considering WINNER model for log normal shadowing with standard deviation $8 \mathrm{~dB}[18]$.

4.5.3. Fast Fading. We are considering urban ITU Pedestrian B channel model with 6 taps [19]. Table 4 describes the complete set of channel between BS and RS. FRS refers to fixed relay station, MRS refers to mobile relay station and UT refers to user terminal/Mobile.

4.6. Relay Selection Algorithm. In this architecture, a UE (User Equipment/Mobile Station) has two choices to receive signals: either from the BS or from one of the relays [20]. A specific algorithm needs to be established to determine from which node (BS or one of the relays) a UE will receive its signal. An SINR-based relay selection algorithm and path-loss-based relay selection algorithms are explained and compared through simulation. In our relay selection algorithms, we assume that all of fixed relays are placed in strategic locations with good receiving signals from the BS.

4.6.1. SNR-Based Selection Algorithm. SINR-based algorithm has two steps.

(1) Out of the " $n$ " relays $\left(R_{1}, \ldots, R_{n}\right)$ in the cell in which the UE resides, select two that are the closest to the UE $\left(R_{1}, R_{2}\right)$.

(2) Compute the SINR between the two closest relays and the UE, and between the BS and the UE.

The node (relay or BS) with the maximum SINR will be responsible for transmitting signals for the UE

$$
R^{(k)} \equiv \arg \max _{i=0: N_{R}}\left(\mathrm{SNR}_{i}^{(k)}\right),
$$

where $\mathrm{SNR}_{i}^{(k)} \equiv \mathrm{SNR}$ measured between relay $i$ and user $k$, index 0 refers to the base station, and $N_{R}$ is total number of relays (fixed + mobile).

4.6.2. Path-Loss-Based Selection Algorithm. We take into consideration lognormal shadowing in addition to distancebased attenuation. The path-loss algorithm has two steps.

(1) Out of the six relays in the cell in which the UE resides, select two that are the closest to the user $\left(R_{1}\right.$, $\left.R_{2}\right)$.

(2) Compute the path-loss between the two closest relays and the UE, and between the BS and the UE.
Base station and relay node use different power level. The node (relay or BS) with the least path-loss will be responsible for transmitting signals for that UE

$$
R^{(k)} \equiv \arg \min _{i=0: N_{R}}\left(\mathrm{PL}_{i}^{(k)}\right)-\psi,
$$

where $\mathrm{PL}_{i}^{(k)} \equiv$ path loss measured between relay $i$ and user $k$, index 0 refers to the base station, $N_{R}$ is the total number of relays (fixed + mobile),

$$
\psi=10 \log _{10}\left(\frac{\mathrm{PL}_{\mathrm{BS}}}{\mathrm{PL}_{\text {relay }}}\right) .
$$

\section{Performance Metrics and Results}

In this paper, we consider the hexagonal deployment collection of cells corresponding to several tiers around the central cell. The number of tiers is parametric and selected at the beginning of simulation. Simulation runs in combined snapshot mode in which mobiles are randomly deployed at each TTI and simulation runs and results are collected for each TTI and average is performed. Fixed and Mobile relays coexist within the cell. Total mobiles are 100 and mobile deploy randomly in each cells. Number of mobiles is constant over a geographical area and deployment is random. We deployed 6 fixed relay in each cell and they are located 2/3 of the cell radius which are parametric. Power of BS is $12 \mathrm{dBW}$ and power of fixed relay is $10 \%$ of BS ( $2 \mathrm{dBW})$.

Mobile relay can be user terminal that can be configured to act as relay. User terminal $n$ is selected to be relay with probability $p$ (for simulation $p=0.5$ ) which is parametric, then the expected number of mobile relays are $N_{r}=N_{u} * p$ ( $N_{u}$ is the number of users terminal and $N_{r}$ is the mobile relays) then the expected number of end terminals are $N_{e}=$ $N_{u} *(1-p) . N_{e}$ is the end terminal. We are using $1024 \mathrm{FFT}$ size, 5 OFDM symbol per frame, 720 data subcarrier with PUSC scheme with 128 cyclic prefix lengths. The cluster size of the cellular network is taken to be equal to 4 . Seven clusters are deployed, but the data is collected for the innermost cluster, that is, the first tier. Both the BSs and UEs are assumed to use omni-directional antennas. Power control is not considered in this simulation, because when AMC is used, power control does not contribute much towards the throughput increase. Summary of simulation parameter is shown in Table 5.

We consider average thought and average spectral efficiency as output metrics, which is calculate as follows. 


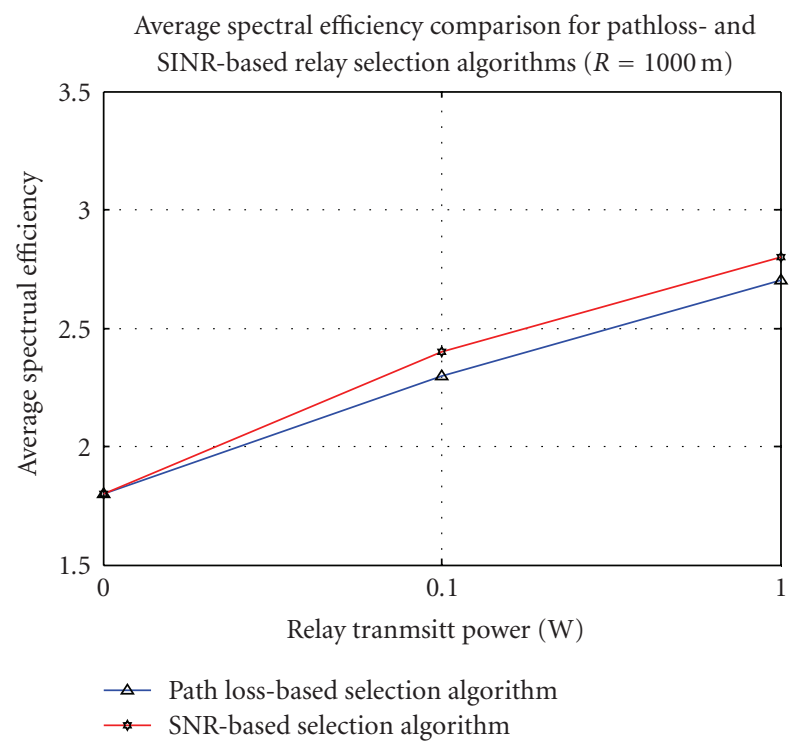

(a)

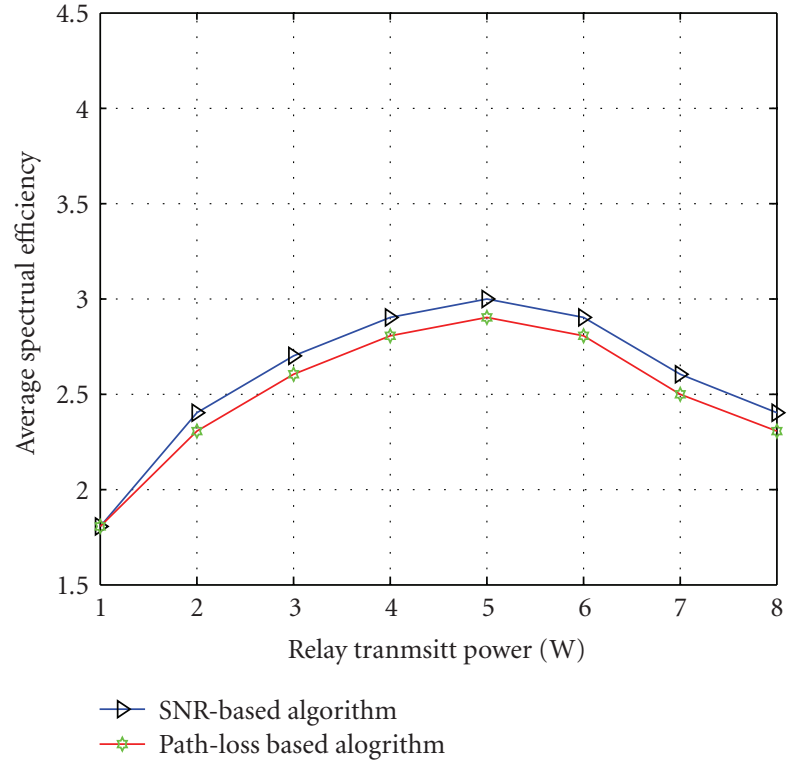

(b)

Figure 9: (a) Path-loss versus SINR-based algorithm. (b) Path-loss versus SINR-based algorithm.

5.1. Service Throughput $S(i, j)$ and OTA (over-the-air) Throughput OTA $(i, j)$. At the end of a given iteration $i$ of the simulator, the OTA throughput and Service Throughput for sector $j$ are computed. OTA $(i, j)$ is defined as the ratio of the number of bits transmitted in sector $j$ over the time required to transmit them.

5.2. Average OTA and Average Service Throughput (per Cell). Obtained by averaging OTA $(i, j)$ and $S(i, j)$ over all sectors and all runs.

5.3. User Service Throughput. This is defined as the number of correctly received bits per user divided by the simulation duration.

5.4. Average Spectral Efficiency. It equals to total number of bit transfer/total time/bandwidth (bit/sec/Hz).

As a performance metric of the network coverage property, the outage probability can be expressed by

$$
P_{o}=P_{r}\left[\gamma<\gamma_{o}\right]=P_{r}[\mathrm{MCS}=0],
$$

where, $\gamma_{0}$ denotes the minimum SINR requirement for the receiver to obtain service, which is set to $0 \mathrm{~dB}$ in this paper. The outage threshold is set to $10 \%$, because full traffic load is adopted in our simulation and the resulting outage probability tends to be overestimated.

In this simulation, we compare the with-relaying case with the without-relaying one. For the without-relaying case, all UEs will receive signals from the BS. In this case the interference comes from the other six BSs. For the withrelaying case, as described above, six relays are placed in each cell. In this case, the received signal can be either from the BS or from a relay depending on which node the UE
TABle 5: Simulation parameters.

\begin{tabular}{|c|c|}
\hline Parameter Name & Value \\
\hline \multicolumn{2}{|l|}{ WiMAX system } \\
\hline $\begin{array}{l}\text { Time transmission interval } \\
\left(T_{\mathrm{ti}}\right)\end{array}$ & $10 \mathrm{~ms}$ \\
\hline Cell type & Omni or TriSector \\
\hline Cell radius & $100,200, \ldots, 1000 \mathrm{~m}$ \\
\hline $\begin{array}{l}\text { Radio Resource } \\
\text { Management }\end{array}$ & Best select CQI \\
\hline Nominal bandwidth $(W)$ & $10 \mathrm{MHz}$ \\
\hline Base Station Power & $10 \mathrm{~W}$ \\
\hline Relay Node Power & $1 \mathrm{~W}, 0.1 \mathrm{~W}$ or $0.3 \mathrm{~W}$ \\
\hline Simulation type & Downlink \\
\hline SINR method & $\begin{array}{l}\text { EESM (Exp Effective SINR } \\
\text { Mapping) }\end{array}$ \\
\hline Channel Model & Urban \\
\hline Carrier frequency & $5 \mathrm{GHz}$ \\
\hline Channel model & ITU vehicular A \\
\hline Mobile terminals velocity & $30 \mathrm{~km} / \mathrm{h}$ \\
\hline Number of Users & 100 per cell \\
\hline Modulation and Coding & $\mathrm{AMC}$ \\
\hline Traffic model & $\begin{array}{l}\text { web3GPP full Queue Option Cell } \\
\text { Arrival Rate: } 10 \text { user/cell/Sec }\end{array}$ \\
\hline Propagation model & $\begin{array}{l}\text { Multipath channel model Path loss, } \\
\text { shadowing and fast fading }\end{array}$ \\
\hline Antenna gain & $14 \mathrm{dBm}$ \\
\hline Duration call & $90 \mathrm{~s}$ \\
\hline Duplex mode & TDD (half duplex) \\
\hline
\end{tabular}



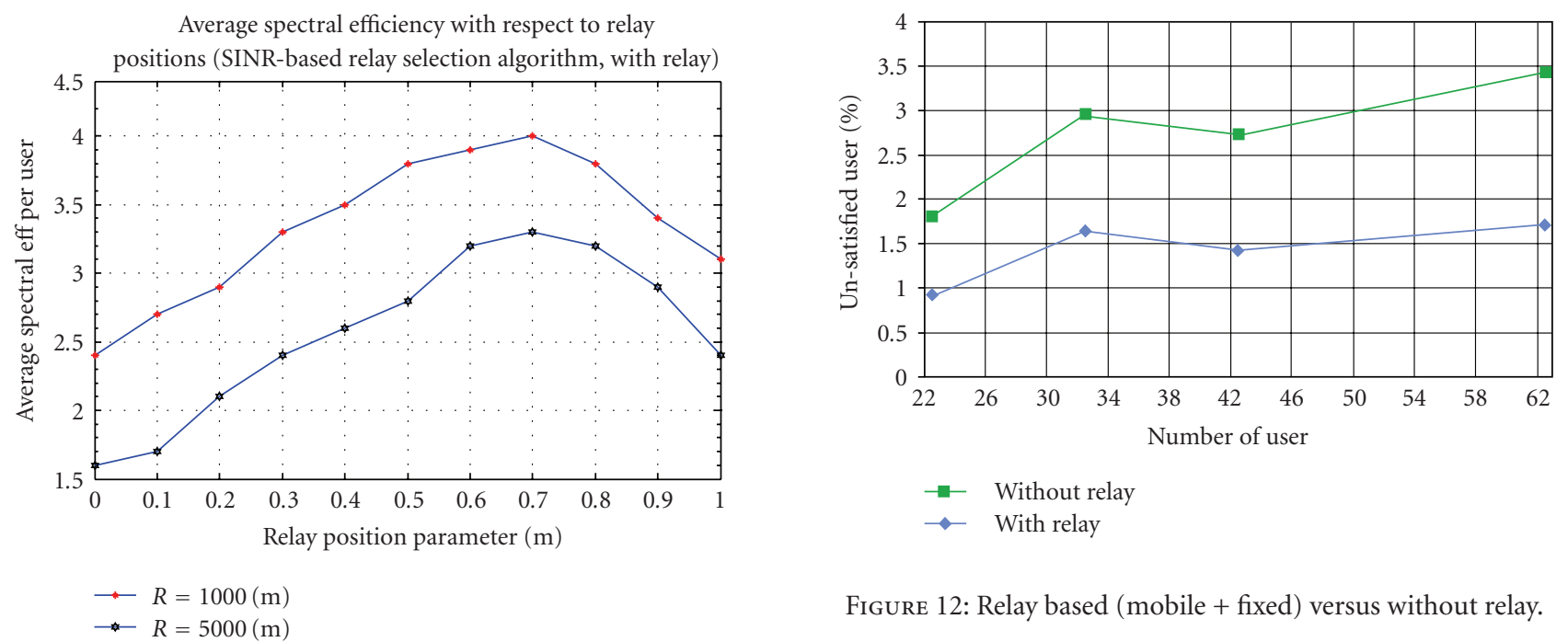

Figure 12: Relay based (mobile + fixed) versus without relay.

FIgURE 10: Relay position with respect to BS.
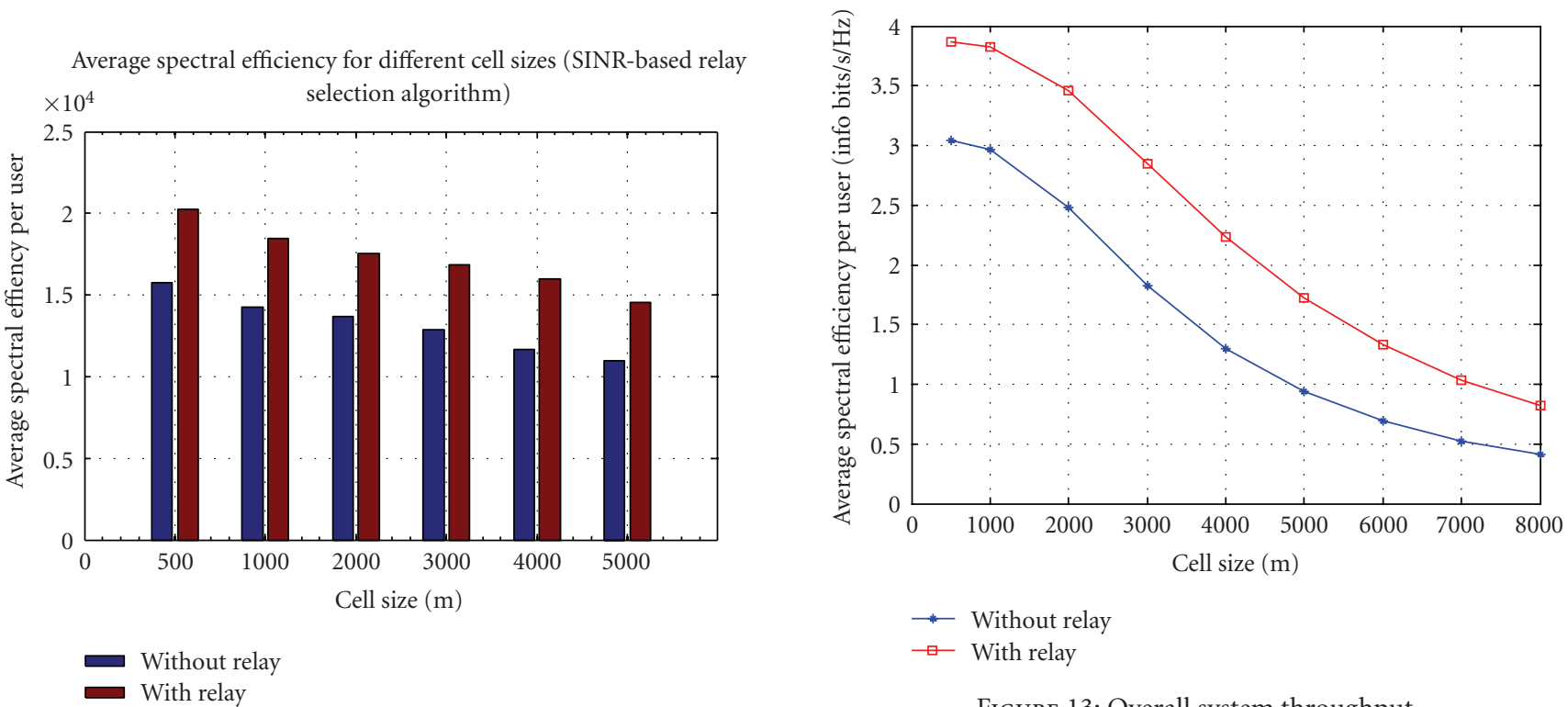

FIGURE 11: Average spectral efficiency for different cell size (central cell).

is communicating with. Figure 9 (a) compares the average throughput for the two relay selection algorithms, path-loss and SINR, without considering the interference from other cells (central cell approach). We can see that SNR-based algorithm is slightly better than path-loss-based algorithm.

Figure 9(b) compare the same algorithm, but this time we consider the interference from other cell (i.e., relay in other cells).

The relay position is indicated by a parameter $m$ showing how far away the relay is from the BS $m=a / R$ where $a$ is the distance from the BS to the relay, $R$ is the distance from the BS to one corner of the cell. In the simulation, the following $m$ values are examined: $0,0.1,0.2 \ldots$ In addition, the results correspond to the $m$ values that are $2 / 3,3 / 4$ and

7/8 are also given out in Figure 10. The results for the $m=0$ case corresponds to the no relay case. The simulation results show that the best range for the relay position is $2 / 3$ to $3 / 4$ away from the BS, when the transmit power ratio of the BS and the relay is $10: 1$. It agrees with our relay position design.

In Figure 11, an average spectral efficiency of $1.7 \mathrm{bits} / \mathrm{sec} / \mathrm{Hz}$ can only be achieved in a small cell $(500 \mathrm{~m})$ without relays, while the same amount of average spectral efficiency can be achieved in a medium cell $(1000 \mathrm{~m})$ with the help of relays. In other words, the system coverage with relays is four times as much as the coverage without relays, because the coverage area is proportional to the square of the radius.

Figure 12 shows the case where fixed and mobile relay coexist in the cell. The percentage of unsatisfied users, by 


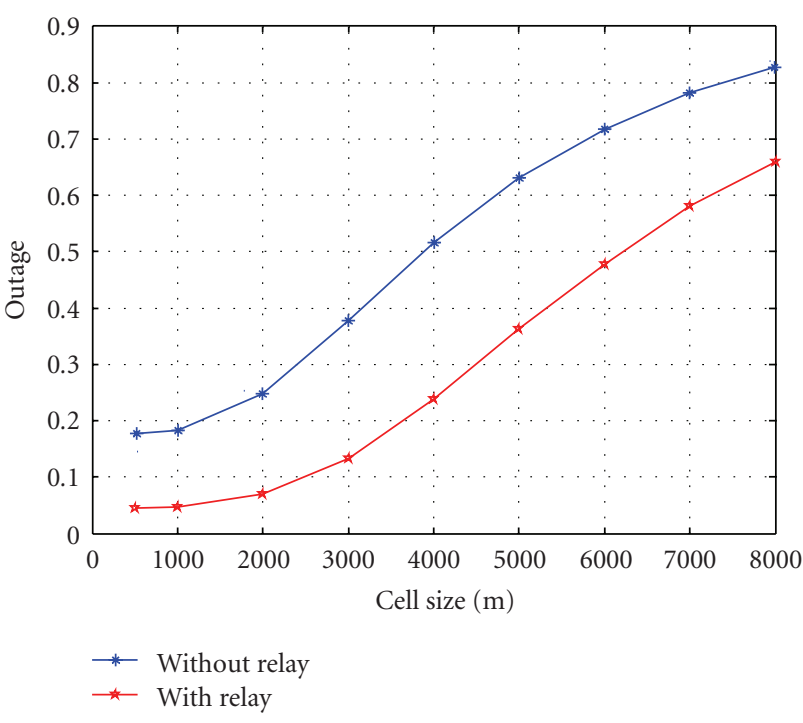

FIgURE 14: Outage probability of system.
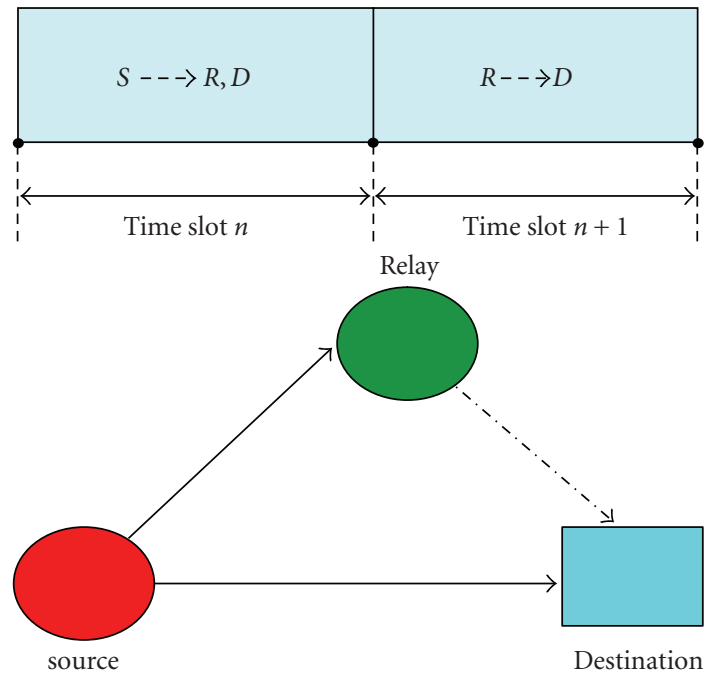

FIgURE 15: Half duplex.

deploying fixed and mobile relay decrease as compared to without relaying case

percentage of unsatisfied user $=\frac{n: \text { drop users }}{m: \text { total number of users }}$

$$
n=\left\{\begin{array}{c}
\text { Packet cannot tranfer " } k \text { " time } \\
\text { OR } \\
\text { SINR }<-10.15 \mathrm{db}
\end{array}\right\} \text {, }
$$

" $k$ " is total number of time packet transfer $(k=5)$ then we consider packet is dropped.

Figure 13 shows the system throughput increase with the relay and outage is decrease as shown in Figure 14.

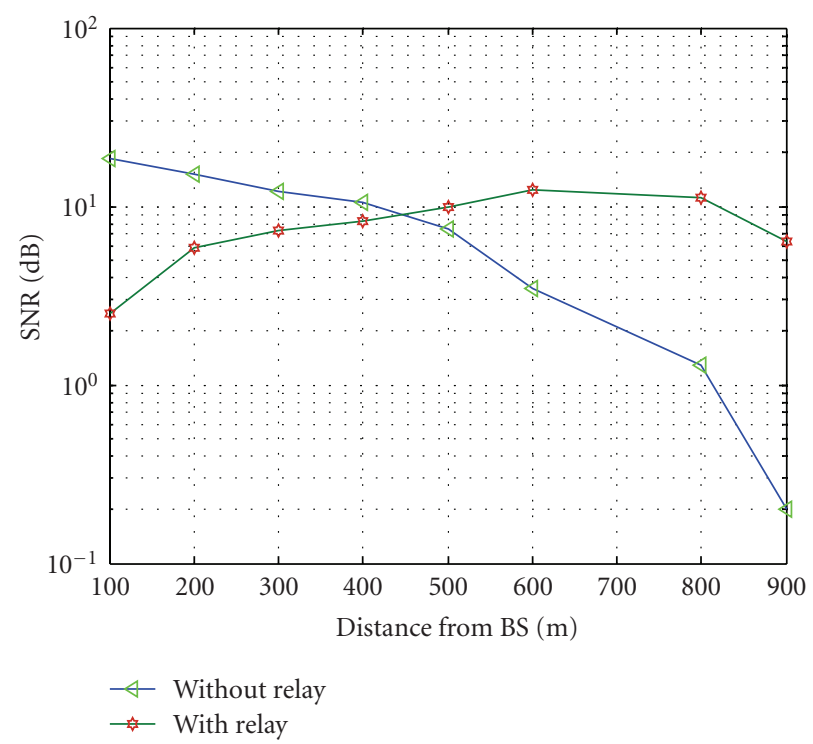

FIGURE 16: Half duplex versus direct link.

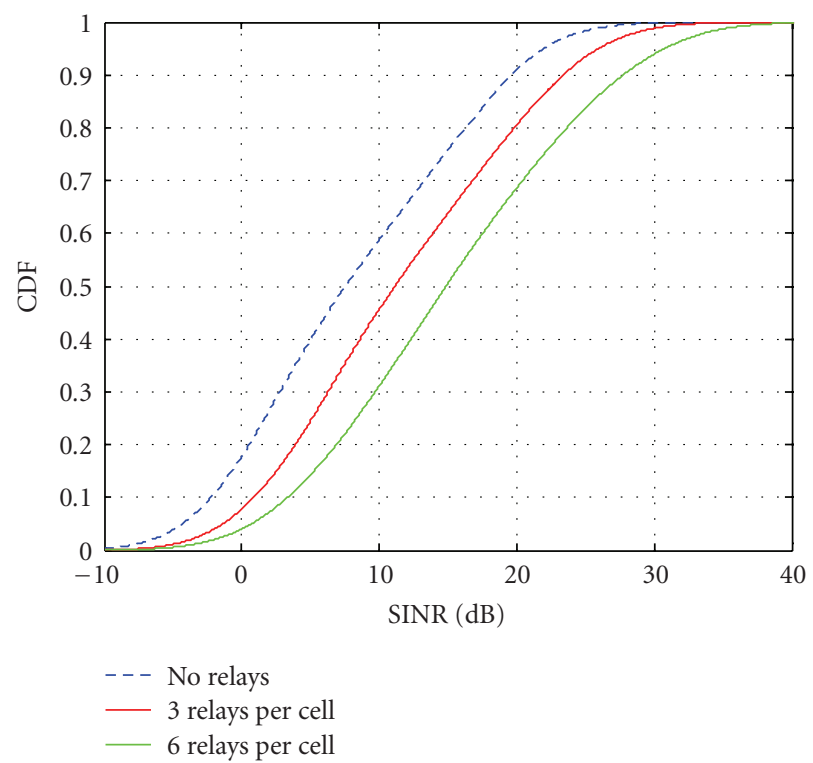

FIGURE 17: SINR at the edge of cells.

We also consider the case of half duplex, where we have two phases as show in Figure 15.

In the simulation results as shown in Figure 16, we observe direct link is better when we have a higher SNR values and when our cell radius start increasing, then half duplex perform better (low SNR).

Figure 17 shows the performance at the edge of the cells. With no relays, we see that $20 \%$ of users are below the threshold ( $\mathrm{SNR}=0 \mathrm{db}$, not communicating with base station), as we increasing the number of relays, the number of users not communicating also decrease (with 3 relay only $8 \%$ user are below the threshold). 

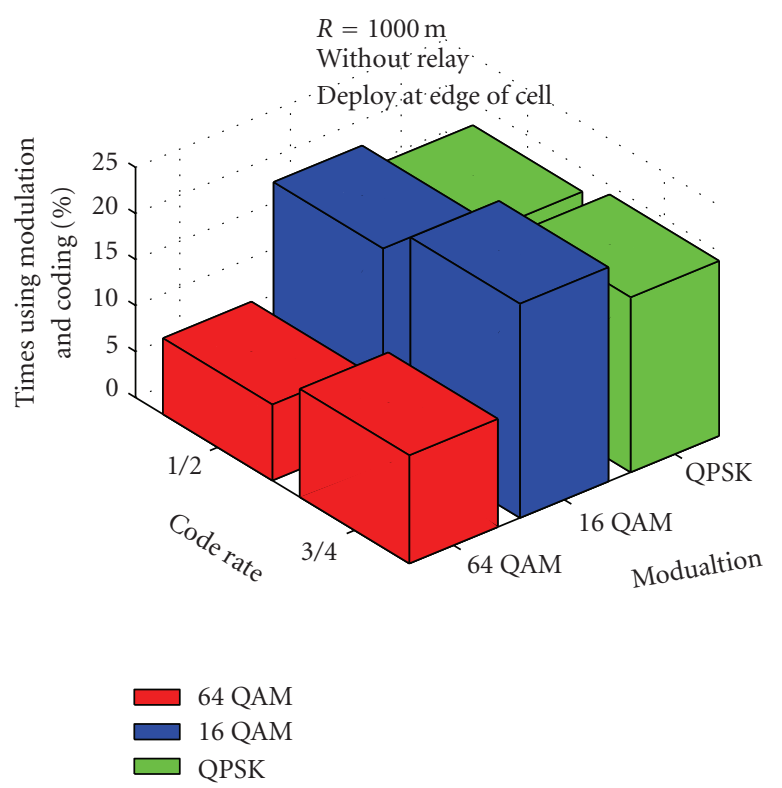

FIgURE 18: Combination of modulation and coding (without relays).

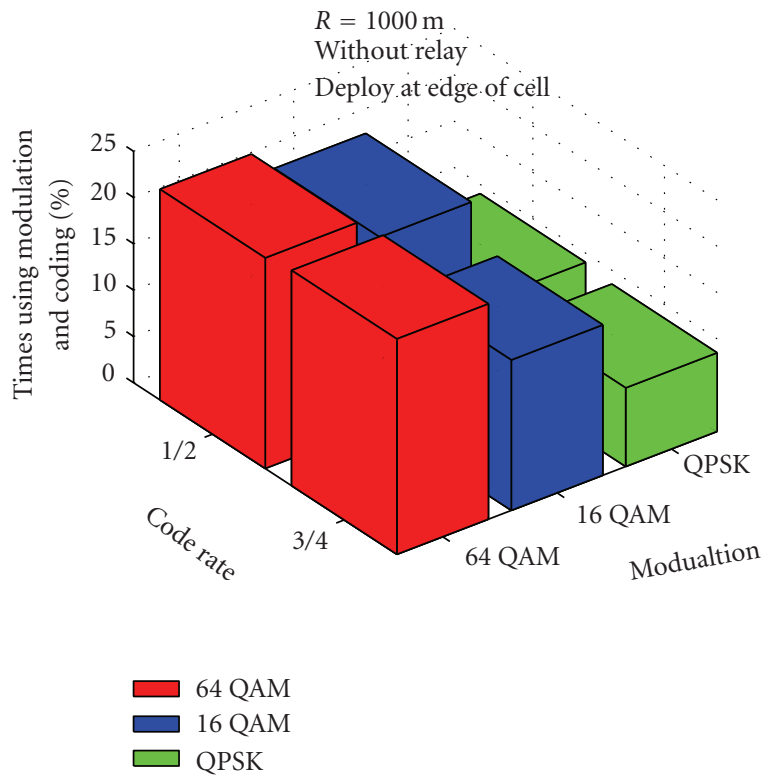

FIGURE 19: Combination of modulation and coding (with relays).

Figures 18 and 19 show how often various combinations of the modulation and coding schemes are used. The results are shown for $1000 \mathrm{~m}$ cells: in figures with relaying case, the combinations of 64-QAM with different code rates are used more often than they are in the without relaying case; this is due to the help provided by relays. In these figures, "percentage of MCS" means percentage of usres whose received SINR value is greater than $4 \mathrm{~dB}$ so that it can support the combination of QPSK and Rate 1/2, which yields the lowest throughput. This value can be regarded as the coverage performance with the required received signal threshold of $4 \mathrm{~dB}$. On the contrary, "\% of failing links" means percentage of usres whose received SINR is less than $4 \mathrm{~dB}$ which corresponds to zero throughput.

\section{Conclusions}

System level simulator is an important tool to evaluate the performance of whole network. In this paper, we covered the important aspects related to the modelling of system level simulator for the relayed-assisted OFDMA. Deployment concepts using relay-assisted OFDMA network have been shown to be of high benefit to substantially reduce the cost of BS to the fixed network (owing to a substantial reduction of $\mathrm{BS}$ needed). Because the envisioned future cellular network cannot cover the same radio range as today's cellular network, it is necessary to deploy relays for radio range extension.

And it is maybe the only way to cover those otherwise shadowed area. Relays have been proven to substantially extend the radio coverage of a BS, especially in highly obstructed service areas. In addition, introducing relays to cellular networks can reduce transmission power for mobile users and offer additional diversity for improving the end-toend radio links. From the simulation results, we can conclude that relaying is a simple yet effective approach to improve system throughput and high data rate coverage.

\section{References}

[1] E. C. van der Meulen, "A survey of multi-way channels in information theory," IEEE Transactions on Information Theory, vol. 23, no. 1, pp. 1-37, 1977.

[2] T. M. Cover and A. A. El Gamal, "Capacity theorems for the relay channel," IEEE Transactions on Information Theory, vol. 25, no. 5, pp. 572-584, 1979.

[3] P. Gupta and P. R. Kumar, "The capacity of wireless networks," IEEE Transactions on Information Theory, vol. 46, no. 2, pp. 388-404, 2000.

[4] A. Sendonaris, E. Erkip, and B. Aazhang, "User cooperation diversity_part I: system description," IEEE Transactions on Communications, vol. 51, no. 11, pp. 1927-1938, 2003.

[5] R. Pabst, B. H. Walke, D. C. Schultz et al., "Relay-based deployment concepts for wireless and mobile broadband radio," IEEE Communications Magazine, vol. 42, no. 9, pp. 8089, 2004.

[6] J. Sydir, "IEEE 802.16 broadband wireless access working group-harmonized contribution on 802.16j (mobile multihop relay) usage models," July 2006.

[7] A. Nosratinia, T. E. Hunter, and A. Hedayat, "Cooperative communication in wireless networks," IEEE Communications Magazine, vol. 42, no. 10, pp. 74-80, 2004.

[8] C. Hoymann and K. Klagges, "MAC Frame concepts to support multi-hop communication in IEEE 802.16 networks," In Wireless Word Research Forum, 2005.

[9] P. Viswanath, D. N. C. Tse, and R. Laroia, "Opportunistic beamforming using dumb antennas," IEEE Transactions on Information Theory, vol. 48, no. 6, pp. 1277-1294, 2002.

[10] ITU-R M.1225, 1997.

[11] Y. Li and H. Xiaojing, The Generation of Independent Rayleigh Faders, Motorola Austaralian Research Center, Sydney, Australia. 
[12] X. Cai and G. B. Giannakis, "A two-dimensional channel simulation model for shadowing processes," IEEE Transactions on Vehicular Technology, vol. 52, no. 6, pp. 1558-1567, 2003.

[13] F. Wang et al., "3GPP TR IEEE802.16e system performance analysis and simulation results," in Proceedings of the Annual IEEE International Symposium on Personal Indoor and Mobile Radio Communications (PIMRC '05), Berlin, Germany, September 2005.

[14] 3GPP TR 25.892, "Feasibility Study for OFDM for UTRAN enhancement," v1.1.0, March 2004.

[15] IEEE Std 802.16-2004, "IEEE Standard for Local and Metropolitan Area Networks-Part 16: Air Interface for Fixed Broadband Wireless Access Systems," October 2004.

[16] M. Dottling, J. Michel, and B. Raaf, "Hybrid ARQ and adaptive modulation and coding schemes for high speed downlink packet access," in Proceedings of the 13th IEEE International Symposium on Personal, Indoor and Mobile Radio Communications, vol. 3, pp. 1073-1077, September 2002.

[17] V. S. Abhayawardhana, I. J. Wassellt, D. Crosby, M. P. Sellars, and M. G. Brown, "Comparison of empirical propagation path loss models for fixed wireless access systems," in Proceedings of the 61st IEEE Vehicular Technology Conference (VTC '05), pp. 73-77, June 2005.

[18] IST-2003-507581 WINNER D3.4 version 1.0, "Definition and assessment of relay based cellular deployment concepts for future radio scenarios considering 1 st protocol characteristics".

[19] 3GPP R1-030224, Nortel Networks, "Update of OFDM SI simulation methodology".

[20] H. Hu, Performance analysis of cellular networks with digital fixed relays, M.S. thesis, University of Carleton, 2003. 

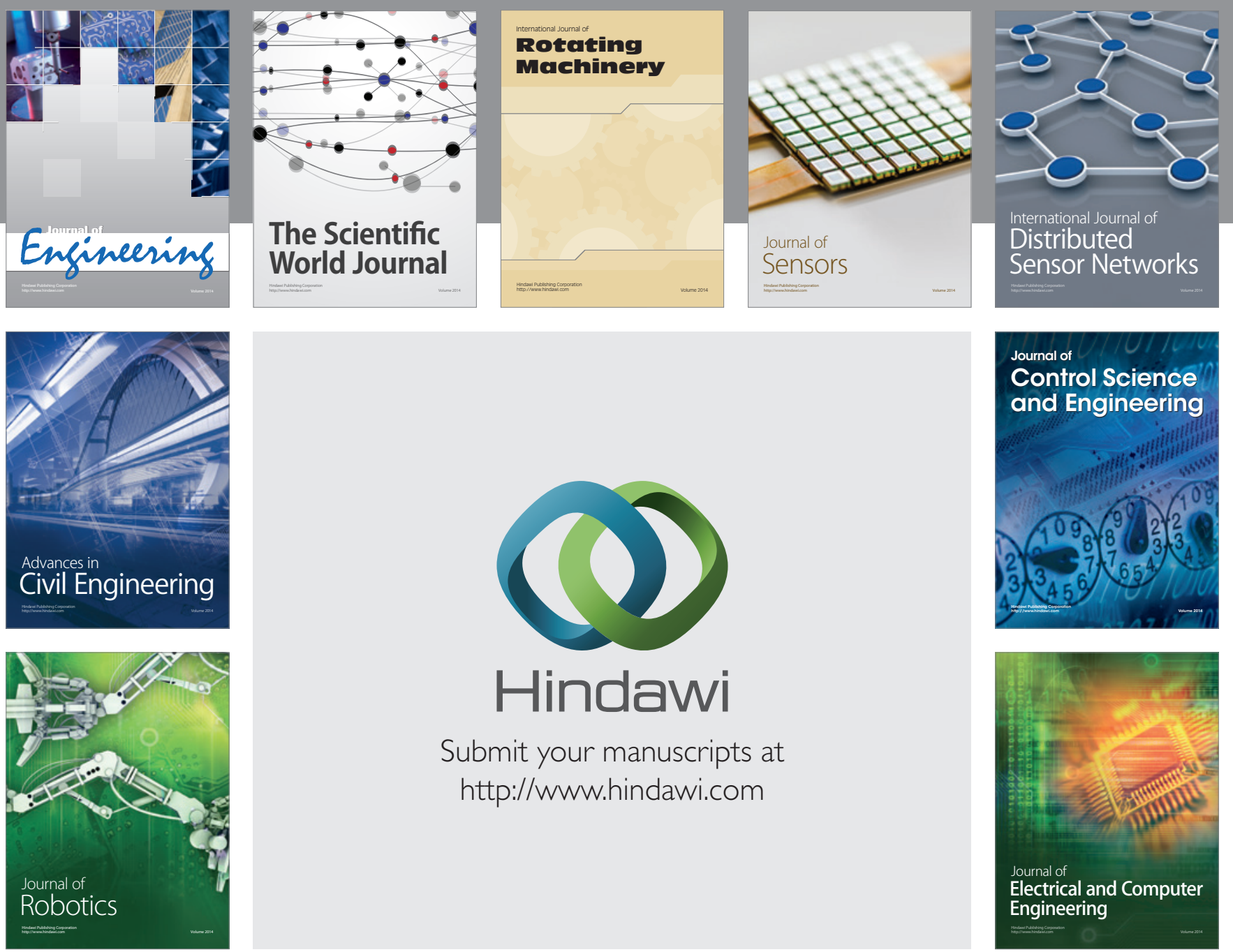

Submit your manuscripts at

http://www.hindawi.com
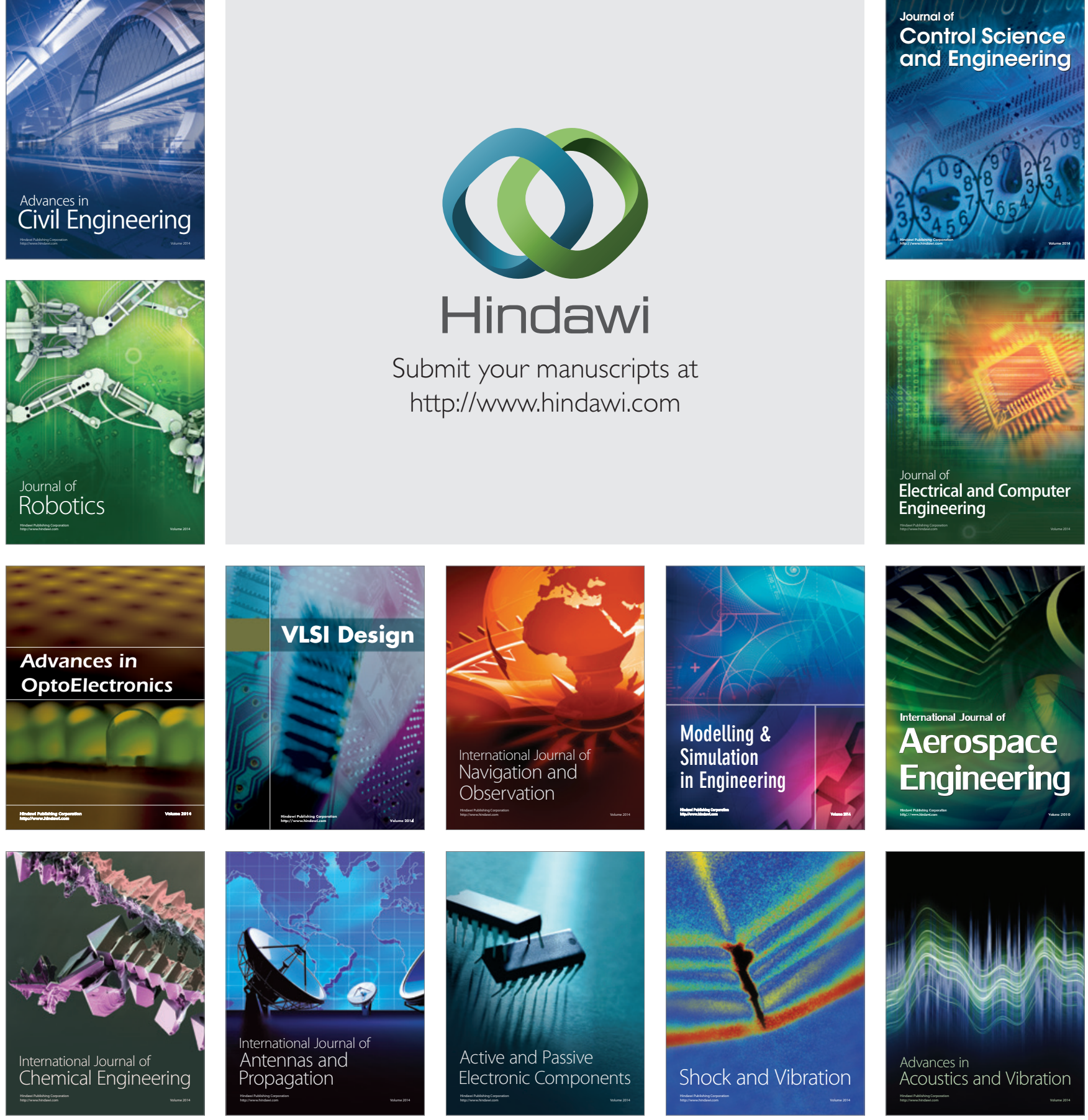